\title{
Growth and Structural Characterization of PbTe/PbEuTe Double Barrier
}

\author{
A. M. P. dos Anjos, E. Abramof, and P. H. O. Rappl \\ Laboratório Associado de Sensores e Materiais, Instituto Nacional de Pesquisas Espaciais, \\ Caixa Postal 515, 12245-970, São José dos Campos-SP, Brazil
}

Received on 4 April, 2005

\begin{abstract}
A series of $\mathrm{PbTe} / \mathrm{PbEuTe}$ double barrier samples with different barrier widths were successfully grown on $\mathrm{BaF}_{2}$ substrates by molecular beam epitaxy. The electron concentration of PbTe spacer and well layers was controlled by the deviation from stoichiometry, while the buffer and cap layers were intentionally doped with bismuth to obtain low-resistivity layers to be used as top and bottom contacts. Assuming 50\% of band offset, a barrier height of $150 \mathrm{meV}$ was determined by infrared transmission measurements, corresponding to a PbEuTe barrier with $5 \%$ of europium content. The structural parameters of the samples were accurately determined by combining the measurement in a high-resolution $\mathrm{x}$-ray diffractometer in the triple-axis configuration with a simulation within the framework of dynamical theory of diffraction.
\end{abstract}

Keywords: $\mathrm{PbTe} / \mathrm{PbEuTe}$ double barrier; $\mathrm{BaF}_{2}$ substrates; Molecular beam epitaxy

\section{INTRODUCTION}

Molecular beam epitaxy (MBE) has been successfully applied for the growth of $\mathrm{PbEuTe} / \mathrm{PbTe}$ heterostructures. New interesting research possibilities were opened by alloying the binary and also ternary lead salts with rare-earth elements to produce compounds with higher energy gaps and their respective multi-layer heterostructures [1-5]. Usually, $\mathrm{PbTe}$ is used for the quantum wells and ternary $\mathrm{Pb}_{1-x} \mathrm{Eu}_{x}$ Te with $\mathrm{x} \leq 0.05$ for the barriers. The advantage of using $\mathrm{Pb}_{1-x} \mathrm{Eu}_{x} \mathrm{Te}$ in lead salt heterostructures is the pronounced increase of the energy band gap with $\mathrm{Eu}$ content. For instance, $\mathrm{Pb}_{1-x} \mathrm{Eu}_{x} \mathrm{Te}$ with $\mathrm{x}=0.06(\mathrm{Eg}=530 \mathrm{meV}$ at $295 \mathrm{~K})$ yields a barrier of 110 $\mathrm{meV}$, considering a band offset of $50 \%[4,5]$.

Transport measurements in $\mathrm{PbTe} / \mathrm{PbEuTe}$ structures have led to interesting results like weak electron localization in $\mathrm{Pb}_{1-x} \mathrm{Eu}_{x}$ Te layers at low temperature [6], observation of the integral quantum Hall effect in $\mathrm{PbTe} / \mathrm{Pb}_{1-x} \mathrm{Eu}_{x} \mathrm{Te}$ multiquantum wells [7], and conductance quantization in small constrictions in PbTe layers [8]. All these transport measurements have been performed with current flowing in the sample plane. To our knowledge, no publication in the literature deals with vertical transport (charge flowing parallel to the growth direction) in IV-VI compounds. The objective of this paper is to grow and characterize the structure of $\mathrm{PbTe} / \mathrm{PbEuTe}$ double barrier samples. These devices are designed to the measurement of resonant tunnelling.

Here, we report on the growth of a series of $\mathrm{PbTe} / \mathrm{PbEuTe}$ double barrier (DB) samples on $\mathrm{BaF}_{2}$ substrates, using a MBE system dedicated to the IV-VI compounds and related europium alloys. The Eu content of the barriers was determined by infrared transmission [5] on reference $\mathrm{Pb}_{1-x} \mathrm{Eu}_{x}$ Te layers. The electrical characteristics of these layers were obtained by Hall effect measurements. In order to determine the structural parameters of the DB's, the X-ray spectra of all samples were measured in a high-resolution diffractometer in the triple-axis configuration.

\section{EXPERIMENTAL AND RESULTS}

The layer sequence of the $\mathrm{PbTe} / \mathrm{PbEuTe}$ double barrier structures, which we intend to grow here, is shown in Fig. 1. The structure is composed of an $n^{+}$-PbTe buffer layer $(\sim 3 \mu \mathrm{m})$ grown on top of a $\mathrm{BaF}_{2}$ substrate, followed by a $14 \mathrm{~nm} n$-PbTe spacer layer, the double barrier itself ( $\mathrm{PbTe}$ well sandwiched between two PbEuTe barriers), another $14 \mathrm{~nm} n$-PbTe spacer, plus a $45 \mathrm{~nm} n^{+}$-PbTe cap layer. The $n^{+}$-PbTe bottom and top layers are to optimize electrical contacts.

All samples were grown on freshly cleaved (111) $\mathrm{BaF}_{2}$ in a Riber 32P MBE system equipped with effusion cells containing solid sources of $\mathrm{PbTe}, \mathrm{Te}(1), \mathrm{Eu}, \mathrm{Te}(2)$ and $\mathrm{Bi}_{2} \mathrm{Te}_{3}$. The first tellurium effusion cell is used to control the stoichiometry of the PbTe layers, while the second one to balance the beam fluxes during the growth of PbEuTe layers. To achieve electron concentration higher than $10^{17} \mathrm{~cm}^{-3}$, it is necessary to dope $\mathrm{PbTe}$ extrinsically through the $\mathrm{Bi}_{2} \mathrm{Te}_{3}$ cell. In a previous work [9], we demonstrated the successful $n$-type doping of $\mathrm{PbTe}$ layers with well-controlled doping levels ranging from $10^{17}$ to $10^{20} \mathrm{~cm}^{-3}$.

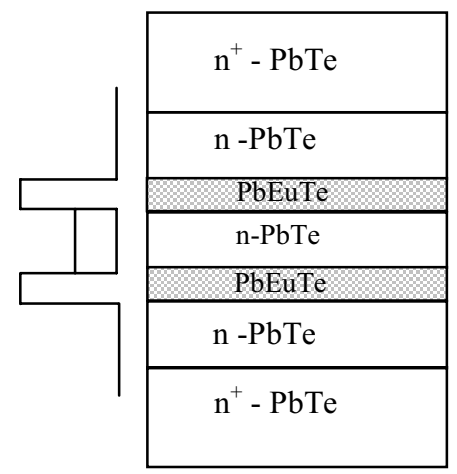

FIG. 1: Layer sequence of a PbTe/PbEuTe double barrier structure.

Before growing the $\mathrm{DB}$ samples properly, $\mathrm{PbTe}$ and $\mathrm{Pb}_{1-x} \mathrm{Eu}_{x}$ Te reference layers were grown to determine the best MBE conditions to achieve the desired layers for the DB struc- 
ture. During this experiment, the PbTe effusion cell temperature was kept fixed at $647^{\circ} \mathrm{C}$, and the layers were always deposited at a substrate temperature of $300^{\circ} \mathrm{C}$. In these conditions the growth rate was about $2 \AA / s$.

The temperature of the $\mathrm{Te}(1)$ effusion cell was adjusted to control the stoichiometry deviation and obtain an $n$-type $\mathrm{PbTe}$ layer with an electron concentration close to $1 \times 10^{17} \mathrm{~cm}^{-3}$. The bismuth telluride beam flux was set to achieve an $n^{+}$$\mathrm{PbTe}$ layer with electron concentration in the range of high $10^{19} \mathrm{~cm}^{-3}$. To determine the electrical properties of the reference layers, Van der Pauw geometry samples were prepared by soldering $\mathrm{Au}$ wires with In pellets. The measurements were performed at 300 and $77 \mathrm{~K}$ in a Keithley Hall effect system with a magnetic field of 7000 Gauss, and the results are summarized in Table 1. To obtain these layers, the $\mathrm{Te}(1)$ and $\mathrm{Bi}_{2} \mathrm{Te}_{3}$ effusion cell temperature was set to 275 and $460{ }^{\circ} \mathrm{C}$, respectively.

Note that the bismuth addition to the $n$-PbTe layer increases the concentration from $1.4 \times 10^{17}$ to $9 \times 10^{19} \mathrm{~cm}^{-3}$ at $77 \mathrm{~K}$ and decrease the resistivity from $1.8 \times 10^{-2}$ to $1.3 \times 10^{-4} \Omega \mathrm{cm}$. The $n^{+}$-PbTe layer is then appropriated for the top and bottom contacts in the DB samples. The carrier mobilities are naturally reduced due to the increase in carrier concentration.

The europium content of the $\mathrm{Pb}_{1-x} \mathrm{Eu}_{x} \mathrm{Te}$ barrier was controlled by the Eu effusion cell temperature and the $\mathrm{Te}(2)$ beam flux is adjusted to maintain a Te/Eu beam flux ratio of 2 during growth. To determine the Eu content, the energy gap of $\mathrm{Pb}_{1-x} \mathrm{Eu}_{x}$ Te reference layers was obtained from infrared transmission spectrum [4,5]. The measurements were performed at $300 \mathrm{~K}$ in a Perkin Elmer FTIR 1600 Fourier transform spectrometer in a frequency range from 500 to about $4500 \mathrm{~cm}^{-1}$ (55 to about $550 \mathrm{meV}$ ). Fig. 2 shows the trans-

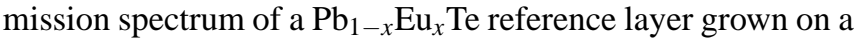
$\mathrm{BaF}_{2}$ substrate with $\mathrm{Eu}$ and $\mathrm{Te}(2)$ effusion cell temperature of 335 and $300^{\circ} \mathrm{C}$, respectively. The energy gap is determined by the cut-off energy at the right-hand side of the spectrum. The Eu content $x$ is then obtained by the following expression [5], which relates the energy gap at $300 \mathrm{~K}$ to the Eu content for $\mathrm{Pb}_{1-x} \mathrm{Eu}_{x}$ Te layers.

$$
E g(m e V)=321+3487.32 x
$$

The Eu content calculated for the reference layer in Fig. 2 was $x=0.05$. This energy gap leads to a potential barrier of $150 \mathrm{meV}$ for PbTe in the DB structure in both conduction and valence bands, assuming 50\% for the band offsets. The electrical properties of this $p$-type $\mathrm{Pb}_{1-x} \mathrm{Eu}_{x} \mathrm{Te}$ reference layer are also shown in Table 1.

After the calibration procedure for the individual layers, the remote MBE shutter control was programmed to obtain the desired double barrier structure. For this series, we decided to vary the PbEuTe barrier thickness, but still keep it below the critical value, in order to maintain the whole DB structure coherent, i.e., with the same in-plane lattice parameter of PbTe. The critical thickness of $\mathrm{Pb}_{1-x} \mathrm{Eu}_{x} \mathrm{Te}$ with $\mathrm{x} \sim 0.05$ on top of $\mathrm{PbTe}$ was evaluated from the simple relation $\mathrm{t}_{c}=1 / f$ (in monolayers) [10], where $f$ is the lattice misfit between both layers. Using 6.460 and $6.473 \AA$ for $\mathrm{PbTe}$ and $\mathrm{Pb}_{0.95} \mathrm{Eu}_{0.05}$ Te lattice

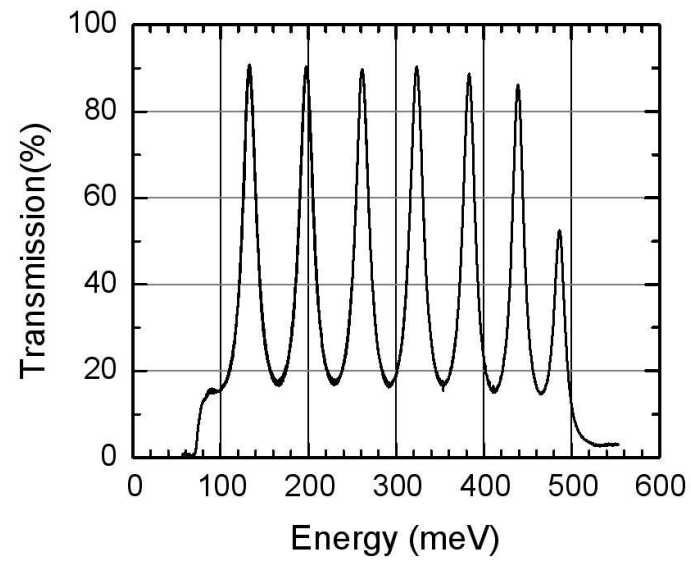

FIG. 2: Infrared transmission spectrum of a $\mathrm{Pb}_{1-x} \mathrm{Eu}_{x}$ Te reference layer. The energy gap is determined by the cut-off at the right side.

constants, respectively, we get a critical thickness of about 180 $\mathrm{nm}$. Considering this top limit, we varied the growth time of $\mathrm{PbEuTe}$ from 15 to $400 \mathrm{~s}$ in our series, keeping the time of $25 \mathrm{~s}$ for the growth of PbTe well.

In situ RHEED at $12 \mathrm{keV}$ was performed in order to evaluate the surface and interface quality of the films during epitaxial deposition. The RHEED transitions from islands to layerby-layer growth mode occurred at about $50 \mathrm{~nm}$ of growth. For the heteroestructure on top of $n$-PbTe buffer, the RHEED pattern remained totally streaked during the entire double barrier growth process, indicating the persistence of a smooth surface throughout the stack.

In order to determine the structural parameters of the $\mathrm{PbTe} / \mathrm{PbEuTe}$ double barrier, the $\mathrm{x}$-ray spectra of all samples were measured in a high-resolution diffractometer in the triple-axis configuration. Our Philips X'Pert diffractometer was configured with a four-crystal $\mathrm{Ge}(220)$ monochromator in the primary optics (between the $\mathrm{Cu}$ x-ray tube and the sample) and a $\mathrm{Ge}(220)$ channel-cut analyzer immediately before the detector to increase substantially the resolution in the $2 \theta$ direction.

Figure 3 shows the $\omega / 2 \theta$ scan around the (222) Bragg diffraction peak for three $\mathrm{PbTe} / \mathrm{PbEuTe} \mathrm{DB}$ samples with different barrier thickness. The spectra shows the $n^{+}$-PbTe buffer layer as the most intense peak, the $\mathrm{BaF}_{2}$ substrate peak, which was used as a reference for the $\Theta$ scale, and the interference pattern form the DB structure itself. Note that the intensity of the diffraction pattern due to the DB structure is two to four orders of magnitude lower than that of the PbTe buffer layer. For this reason, we had to use a large integration time $(5 \mathrm{~s})$ for each angular step. Observe also that, as the barrier width increases, the most intense peak of the DB diffraction pattern moves to lower angles away from the PbTe buffer peak, and increases in intensity.

To obtain the structural parameters of the DB samples, the spectra were calculated within the framework of the dynamical theory of $\mathrm{x}$-ray diffraction using Takagi-Taupin equations 
TABLE I: Resistivity $(\rho)$, Hall mobility $(\mu)$ and carrier concentration $(n / p)$ for individual layers at 77 and $300 \mathrm{~K}$.

\begin{tabular}{|l|l|l|l|l|}
\hline \multicolumn{1}{|c|}{ Layer } & $\begin{array}{c}\mathrm{T} \\
(\mathrm{K})\end{array}$ & \multicolumn{1}{c|}{$\begin{array}{c}\rho \\
(\Omega \mathrm{cm})\end{array}$} & $\begin{array}{c}\mu \\
\left(\mathrm{cm}^{2} / \mathrm{Vs}\right)\end{array}$ & $\begin{array}{c}n / p \\
\left(\mathrm{~cm}^{-3}\right)\end{array}$ \\
\hline$n$-PbTe & 77 & $1.86 \times 10^{-2}$ & 23100 & $1.45 \times 10^{17}$ \\
& 300 & $1.01 \times 10^{-1}$ & 459 & $1.33 \times 10^{17}$ \\
\hline$n^{+}-\mathrm{PbTe}$ & 77 & $1.34 \times 10^{-4}$ & 514 & $9.02 \times 10^{19}$ \\
& 300 & $3.94 \times 10^{-4}$ & 195 & $8.10 \times 10^{19}$ \\
\hline$p$ - $\mathrm{Pb}_{1-x} \mathrm{Eu}_{x} \mathrm{Te}$ & 77 & $1.72 \times 10^{-1}$ & 113 & $3.18 \times 10^{17}$ \\
$\mathrm{x}=0.05$ & 300 & $3.51 \times 10^{-1}$ & 38 & $4.61 \times 10^{17}$ \\
\hline
\end{tabular}
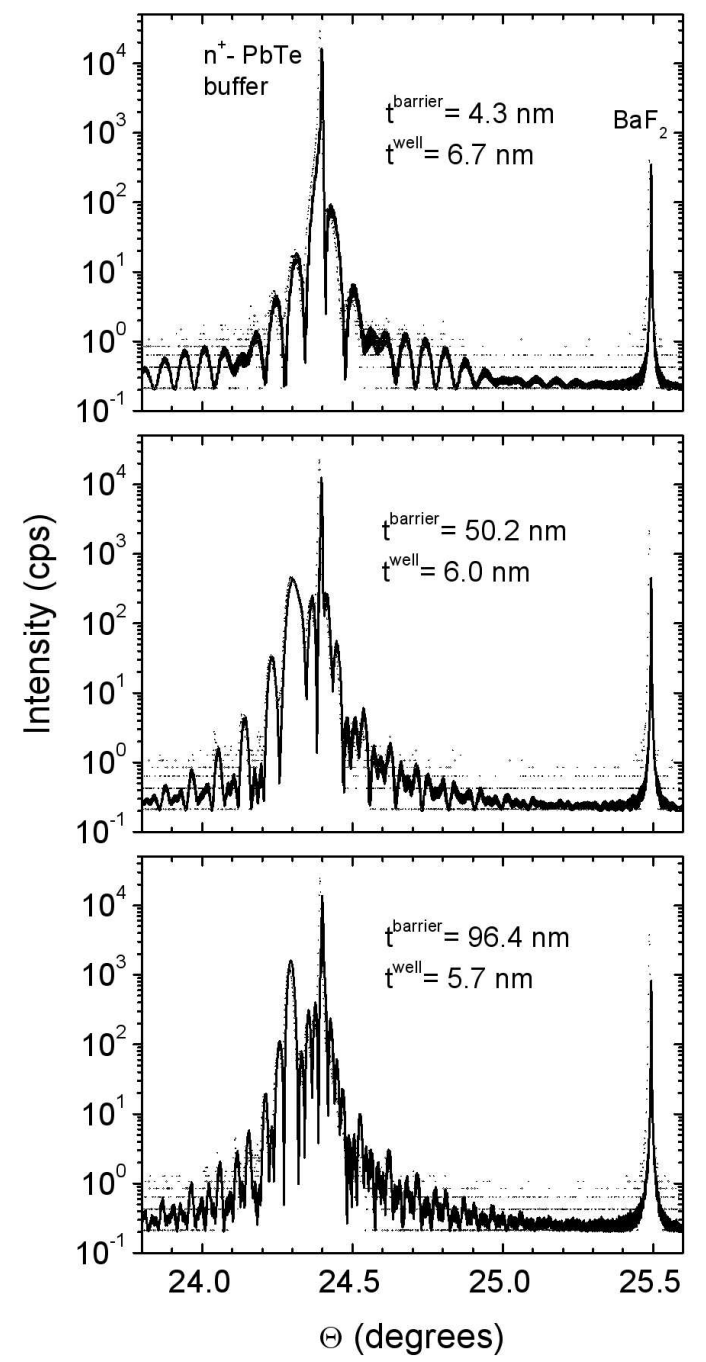

FIG. 3: $\omega / 2 \Theta$ scans around the (222) Bragg diffraction peak of three $\mathrm{PbTe} / \mathrm{PbEuTe}$ double barrier samples, with different barrier thicknesses, grown on $\mathrm{BaF}_{2}$ substrates.
TABLE II: Double barrier structural parameters obtained from the $\mathrm{x}$ ray analysis: lattice parameter (a) of PbTe and PbEuTe and thickness $(\mathrm{t})$ of the well (W), barrier (B), spacer (S), and cap (C) layers.

\begin{tabular}{|c|c|c|c|c|c|c|}
\hline Sample & \multicolumn{2}{|c|}{$\mathrm{a}(\AA)$} & \multicolumn{4}{c|}{$\mathrm{t}(\AA)$} \\
\hline & PbTe & PbEuTe & W & B & S & C \\
\hline 4050 & 6.4600 & 6.4741 & 60 & 45 & 144 & 432 \\
\hline 4019 & 6.4603 & 6.4743 & 67 & 43 & 162 & 486 \\
\hline 4100 & 6.4608 & 6.4750 & 57 & 242 & 138 & 414 \\
\hline 4099 & 6.4607 & 6.4735 & 60 & 502 & 144 & 432 \\
\hline 4098 & 6.4600 & 6.4734 & 57 & 964 & 138 & 414 \\
\hline
\end{tabular}

\section{$[3,11]$ and compared to the measured $\omega / 2 \theta$ scans.}

For the simulation, we take the sample structure shown in Fig. 1 and assumed that the PbEuTe barriers are completely strained to the PbTe layer, which is confirmed by the bell shape of the diffraction pattern. First of all, the lattice constant of PbTe is adjusted to fit both PbTe buffer and $\mathrm{BaF}_{2}$ $(a=6.2000 \AA)$ substrate peaks. The value found for the PbTe lattice constant for each sample is shown in Table 2. A mean value of $6.4604 \AA$ is encountered. In the fitting program, the thickness of each individual layer was calculated by the growth rate multiplied by the respective MBE shutter opening time. The PbEuTe growth rate was considered to be $(1+\mathrm{x})$ times the $\mathrm{PbTe}$ growth rate. In this manner, the $\mathrm{PbTe}$ growth rate and the PbEuTe lattice constant remained as the main fitting parameters. The values found for the PbEuTe relaxed lattice constant are also shown in Table 2.

The simulation results gave a value of $2.40 \pm 0.15 \AA / s$ for the $\mathrm{PbTe}$ growth rate. The thickness obtained for the individual (well, barrier, spacer and cap) layers of each DB sample are summarized in Table 2. The well width was 6 to $7 \mathrm{~nm}$ while the barriers thickness varied from 4 to $96 \mathrm{~nm}$. Even for the DB sample with the widest barrier, the assumption of pseudomorphic structure fitted well the measured x-ray spectrum, as shown in the lower panel of Fig. 3, and expected from the estimated critical thickness. The measured spacer thickness was $14 \mathrm{~nm}$ and the cap layer thickness was found to be 42 to $49 \mathrm{~nm}$. The results obtained from the x-ray analysis indicated that the control on our molecular beam epitaxial system is appropriated to produce the desired double barrier structures shown in Fig. 1. 


\section{CONCLUSION}

We have successfully produced a series of $\mathrm{PbTe} / \mathrm{PbEuTe}$ double barrier structures, with different barrier thickness, grown on $\mathrm{BaF}_{2}$ substrates by molecular beam epitaxy. The electron concentration of the $\mathrm{PbTe}$ spacer and well layers was controlled by the deviation from stoichiometry, while the buffer and cap layer were intentionally doped with bismuth to obtain low-resistivity layers to be used as top and bottom contacts. It was possible to have a good control on the europium content of the PbEuTe layers. A value of $x=0.05$ was chosen for this series. The x-ray analysis allowed an accurate determination of the structural parameters, and the agreement between the measured and calculated x-ray spectra indicated that coherent double barrier structures with sharp interfaces were obtained.

\section{Acknowledgement}

We are grateful to partial support of FAPESP (Grant $\mathrm{Nr}$. 00/12529-6) and CAPES (scholarship of first author).
[1] G. Springholz and G. Bauer, J. Cryst. Growth 144, 157 (1994).

[2] D. L. Partin, IEEE J. Quantum Electron. QE-24, 1716 (1988).

[3] E. Abramof, P. H. O. Rappl, A. Y. Ueta, and P. Motisuke, J. Appl. Phys. 88, 725 (2000).

[4] E. Abramof, E. A. de Andrada e Silva, S. O. Ferreira, P. Motisuke, P. H. O. Rappl, and A. Y. Ueta, Phys. Rev. B 63, 085304 (2001).

[5] S. Yuan, H. Krenn, G. Springholz, A. Y. Ueta, G. Bauer, and P. J. McCann, Phys. Rev. B. 55, 4607 (1997).

[6] A. Prinz, G. Brunthaler, A. Y. Ueta, G. Springholz, G. Bauer, G. Grabecki, and T. Dietl, Phys. Rev. B 59, 12983 (1999).

[7] G. Sprinholz, G. Ihninger, G. Bauer, M. M. Olver, J. Z. Pasta- lan, S. Romaine, and B. B. Goldberg, Appl. Phys. Lett. 63, 21 (1993).

[8] G. Grabecki, J. Wróbel, T. Dietl, K. Byczuk, E. Papis, E. Kaminska, A. Piotrowska, G. Spingholz, M. Pinczolits, and G. Bauer, Phys. Rev. B 60, R5133 (1999).

[9] A. M. P. dos Anjos, E. Abramof, P. H. O. Rappl, A. Y. Ueta, and H. Closs, Braz. J. Phys. 34, 653 (2004).

[10] D. J. Dunstan, S. Young, and R. H. Dixon, J. Appl. Phys. 70, 3038 (1991)

[11] W. J. Bartels, J. Honstra, and D. J. W. Lobeek, Acta Crystallogr., Sect. A: Found. Crystallogr. 45, 539 (1986). 